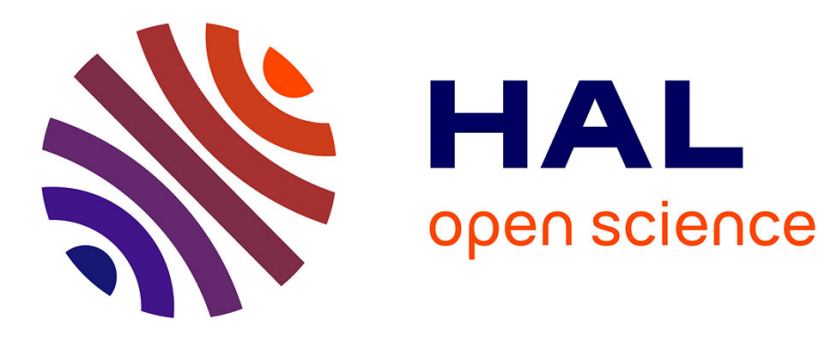

\title{
Copula-Based Interference Models for IoT Wireless Networks
}

Ce Zheng, Malcolm Egan, Laurent Clavier, Gareth Peters, Jean-Marie Gorce

\section{To cite this version:}

Ce Zheng, Malcolm Egan, Laurent Clavier, Gareth Peters, Jean-Marie Gorce. Copula-Based Interference Models for IoT Wireless Networks. ICC 2019 - 53rd IEEE International Conference on Communications, May 2019, Shanghai, China. pp.1-6, 10.1109/ICC.2019.8761783 . hal-02065780

\section{HAL Id: hal-02065780 \\ https://hal.science/hal-02065780}

Submitted on 13 Mar 2019

HAL is a multi-disciplinary open access archive for the deposit and dissemination of scientific research documents, whether they are published or not. The documents may come from teaching and research institutions in France or abroad, or from public or private research centers.
L'archive ouverte pluridisciplinaire HAL, est destinée au dépôt et à la diffusion de documents scientifiques de niveau recherche, publiés ou non, émanant des établissements d'enseignement et de recherche français ou étrangers, des laboratoires publics ou privés. 


\title{
Copula-Based Interference Models for IoT Wireless Networks
}

\author{
Ce Zheng, Malcolm Egan, Laurent Clavier, Gareth W. Peters and Jean-Marie Gorce
}

\begin{abstract}
As the Internet of Things (IoT) is largely supported by wireless communication networks in unlicensed bands, there has been a proliferation of technologies that use a large variety of protocols. An ongoing challenge is how these networks can coexist given that they have different power levels, symbol periods, and access protocols. In this paper, we study the statistics of interference due to IoT networks that transmit small amounts of data. A key observation is that sets of active devices change rapidly, which leads to impulsive noise channels. Moreover, these devices operate on multiple partially overlapping resource blocks. As such, we characterize the joint distribution and propose a tractable model based on copulas. Using our copula model, we derive closed-form achievable rates. This provides a basis for resource allocation and network design for coexisting IoT networks.
\end{abstract}

\section{INTRODUCTION}

With the increasing scale of wireless network deployments for the Internet of Things (IoT), an ongoing challenge is to ensure that these networks can coexist. A key issue is that interference from a large number of devices, even if they operate at low power levels, can degrade the performance of other communication networks. This means that the interference statistics are difficult to characterize and has lead to a number of experimental studies on the interference in various contexts [1]-[4].

One feature observed in IoT networks is the presence of impulsive interference, where high amplitude interference is significantly more likely than in Gaussian models. This behavior has been observed both in experimental studies [4] and also in theoretical analysis [5], [6]. As a consequence, Gaussian models are often not appropriate and the interference statistics lie in a more general class of models.

Introducing non-Gaussian interference models implies that the interference statistics are not simply characterized by their mean and variance. This issue is amplified in settings where a number of frequency bands are used for transmissions. In these cases, the covariance matrix is not sufficient in order to characterize the joint interference statistics over multiple frequency bands. This is particularly evident when the frequency bands used by different users only partially overlap [7], such as in non-orthogonal multiple access (NOMA) schemes [8] including sparse code multiple access (SCMA) [9].

Due to the rich dependence structure possible for the joint distribution of non-Gaussian random vectors, a key question is how it should be modeled. For the purposes of analysis,

Zheng, C. and L. Clavier are with Université de Lille 1, IEMN UMR CNRS 8520, IRCICA USR CNRS 3380, Villeneuve d'Ascq, France. M. Egan and J.M. Gorce are with Univ. Lyon, INSA Lyon, INRIA, CITI, France. L. Clavier is also with IMT Lille Douai, France. G.W. Peters is with the School of Mathematical and Computer Sciences, Heriot-Watt University, UK. simulation and design, it is highly desirable that the class of distributions captures the realistic dependence between the interference on different bands, and admits tractable estimation and simulation methods.

A popular method in statistics for tractably modeling nonGaussian multivariate distributions is based on copulas [10]. Copula models have been applied in wireless communications to model fading in MIMO systems [11], for blind source separation [12], and modeling interference in ultra wideband or ad hoc networks [13], [14].

In the copula modeling approach, the joint distribution function of a random vector in $\mathbb{R}^{n}, \mathbf{X}=\left[X_{1}, \ldots, X_{n}\right]$ is given in the form

$$
F\left(x_{1}, \ldots, x_{n}\right)=C\left(F_{1}\left(x_{1}\right), \ldots, F_{n}\left(x_{n}\right)\right),
$$

where $C:[0,1]^{n} \rightarrow[0,1]$ is called a copula function, and $F_{i}, i=1, \ldots, n$ are the marginal distribution functions. When both the joint and marginal distributions admit density functions, the joint probability density function is then given in the form

$$
p_{\mathbf{X}}\left(x_{1}, \ldots, x_{n}\right)=c\left(F_{1}\left(x_{1}\right), \ldots, F_{n}\left(x_{n}\right)\right) \prod_{i=1}^{n} p_{X_{i}}\left(x_{i}\right) .
$$

That is, the joint probability density function decomposes into the product of the marginal densities and another function $c:[0,1]^{n} \rightarrow \mathbb{R}_{+}$which captures dependence between the different components of $\mathbf{X}$.

In this paper, we introduce copula models for interference in the context of IoT wireless networks. In particular, we adopt the class of $t$-copulas with $\alpha$-stable marginal distributions. This choice of marginals is consistent with theoretical models for interference arising from Poisson spatial fields of interferers [5], [15]-[17] that exhibits heavy-tails and consistent with experimental studies [4].

To show that $t$-copulas are a good fit for the interference dependence structure, we study simulated data sets arising from a Poisson spatial field of interferers and a SCMA scheme. In our model, interferers randomly select frequency bands to transmit on, leading to partial overlaps. Our fits are shown to be close in terms of the Kullback-Leibler (KL) divergence. Since parameters from $t$-copulas can also be efficiently estimated and the resulting random vectors easily simulated, our proposed interference model satisfies several criteria for a desirable model.

In order to emphasize the tractability of working with the $t$-copula model, we also study achievable rates of a communication system with this class of interference. In particular, we consider a system where two devices communicate in 
a point-to-point fashion with interference modeled via the $t$-copula. This setting arises when the active interferer sets change rapidly; that is, on a symbol-by-symbol basis [5]-[7]. Such a setting is realistic when the point-to-point link exploits a different protocol to that of the interferers; for example on the $868 \mathrm{MHz}$ band, where Zigbee coexists with the wide area networks of SigFox and LoRa. We derive limits on the resulting additive noise channel. In particular, achievable rates are derived, which form a tractable basis for further analysis and design of IoT networks.

\section{SySTEM MODEL}

Consider a network of interfering devices, which is observed by a device at the origin. The locations of the interfering devices form a homogeneous Poisson point process with intensity $\lambda$. Each interfering device transmits over a subset of orthogonal frequency bands, $\mathcal{B}=\{1,2, \ldots, K\}$.

At each time $t$, interfering devices independently transmit on a band $k \in \mathcal{B}$, with probability $p>0$ ( $p$ is same for each band). The probability $p$ can be interpreted as proportional to the quantity of data each interfering device seeks to transmit. As such, the probability that an interfering device transmits on $M$ out of $K$ bands is $\left(\begin{array}{l}K \\ M\end{array}\right) p^{M}(1-p)^{K-M}$. Due to independent thinning, the locations of the subset of transmitting devices on band $k$ are also governed by a Poisson point process. We call $\Phi$ the set of interferers, a Poisson point process with intensity $\lambda$, and $\Phi_{k}(t)$ the set of active interferers at time $t$ on frequency band $k$, a Poisson point process with intensity $\lambda p$.

The interference observed at the origin at time $t$ on frequency band $k$ is given by

$$
z_{k}(t)=\sum_{j \in \Phi_{k}(t)} r_{j}(t)^{-\eta / 2} h_{j, k}(t) x_{j, k}(t),
$$

where $r_{j}$ is the distance from device $j \in \Phi_{k}(t)$ to the origin, $\eta$ is the path loss exponent, $h_{j, k}(t)$ is a Rayleigh fading coefficient with $h_{j, k}(t) \sim \mathcal{C N}(0,1)$, and $x_{j, k}(t)$ is the baseband emission.

Suppose that in addition to the set of interferers, there is an interference-limited point-to-point commmunication system consisting of a device located at the origin and a transmitter located at a distance $d$ from the origin. Hence at time $t$, the device at the origin observes the signal on the $k$-th frequency band given by

$$
y_{k}(t)=d^{-\eta / 2} g_{k}(t) w_{k}(t)+z_{k}(t),
$$

where $g_{k}(t)$ is the Rayleigh fading coefficent on the $k$-th band distributed according to $g_{k}(t) \sim \mathcal{C N}(0,1), w_{k}(t)$ is the baseband emission on band $k$ and $z_{k}(t)$ is the interference in (3) on band $k$. In the following, we will focus on the interference limited regime and neglect the thermal noise arising at the receiver.

Motivated by the fact that IoT networks transmit noncontinguously small quantities of data and there exist protocols that have large variations in symbol periods, we assume that the interference at time $t$ is independent of the interference at any other time $t^{\prime}$, corresponding to different symbols of the point-to-point communication system. The main consequence of this assumption is that the interference random vector $\mathbf{z}(t)=\left[z_{1}(t), \ldots, z_{K}(t)\right]^{T}$ is independent in $t$ and hence the additive noise channel in (4) is memoryless and stationary. We remark that although $\mathbf{z}(t)$ is independent in time, in general the elements of $\mathbf{z}(t)$ corresponding to different frequency bands will be dependent.

Due to the additive nature of the channel in (4), it can be viewed as a stationary memoryless real-valued $2 K$ dimensional vector additive noise channel. Note that since the channel is both memoryless and stationary, we drop the time index and write the channel as

$$
\mathbf{Y}=\mathbf{A X}+\mathbf{Z}
$$

where $\mathbf{A}$ is a real diagonal matrix, and $\mathbf{Y}, \mathbf{X}$ and $\mathbf{Z}$ are all random vectors on the measurable space ${ }^{1}\left(\mathbb{R}^{2 K}, \mathcal{B}\left(\mathbb{R}^{2 K}\right)\right)$. The noise vector $\mathbf{Z}$ is given by

$$
\mathbf{Z}=\left[\operatorname{Re}\left(z_{1}\right), \operatorname{Im}\left(z_{1}\right), \ldots, \operatorname{Re}\left(z_{K}\right), \operatorname{Im}\left(z_{K}\right)\right]^{T} .
$$

\section{Copula-BAsed Interference Models}

In this section, we develop our copula-based interference model and validate its use on a simulated data set obtained from the Poisson spatial field of interferers detailed in Section II. As $\alpha$-stable models will play a key role in our analysis, we first recall their properties that will be used in developing our model.

\section{A. $\alpha$-Stable Distribution Preliminaries}

The $\alpha$-stable random variables have heavy-tailed probability density functions, which have been widely used to model impulsive signals [18], [19]. The probability density function of an $\alpha$-stable random variable is parameterized by four parameters: the exponent $0<\alpha \leq 2$; the scale parameter $\gamma \in \mathbb{R}_{+}$; the skew parameter $\beta \in[-1,1]$; and the shift parameter $\delta \in \mathbb{R}$. As such, a common notation for an $\alpha$-stable random variable $X$ is $X \sim S_{\alpha}(\gamma, \beta, \delta)$. In the case $\beta=\delta=0$, $X$ is said to be a symmetric $\alpha$-stable random variable.

In general, $\alpha$-stable random variables do not have closedform probability density functions. Instead, they are usually represented by their characteristic function, given by $[19, \mathrm{Eq}$. 1.1.6]

$\mathbb{E}\left[e^{i \theta X}\right]=\left\{\begin{array}{l}\exp \left\{-\gamma^{\alpha}|\theta|^{\alpha}\left(1-i \beta(\operatorname{sign} \theta) \tan \frac{\pi \alpha}{2}\right)+i \delta \theta\right\}, \alpha \neq 1 \\ \exp \left\{-\gamma|\theta|\left(1+i \beta \frac{2}{\pi}(\operatorname{sign} \theta) \log |\theta|\right)+i \delta \theta\right\}, \alpha=1\end{array}\right.$

It is possible to extend the notion of an $\alpha$-stable random variable to the multivariate setting. In general $d$-dimensional symmetric $\alpha$-stable random vectors are represented via their characteristic function, given by [19]

$$
\mathbb{E}\left[e^{i \boldsymbol{\theta} \cdot \mathbf{x}}\right]=\exp \left(-\int_{\mathbb{S}^{d-1}}\left|\sum_{k=1}^{d} \theta_{k} s_{k}\right|^{\alpha} \Gamma(d \mathbf{s})\right),
$$

where $\Gamma$ is the unique symmetric measure on the $d$ dimensional unit sphere $\mathbb{S}^{d-1}$. A particular class of $\alpha$-stable

${ }^{1} \mathcal{B}\left(\mathbb{R}^{2 K}\right)$ denotes the Borel $\sigma$-algebra of $\mathbb{R}^{2 K}$. 
random vectors are an instance of the sub-Gaussian $\alpha$-stable random vectors ${ }^{2}$, defined as follows.

Definition 1. Any vector $\mathbf{X}$ distributed as $\mathbf{X}=$ $\left(A^{1 / 2} G_{1}, \ldots, A^{1 / 2} G_{d}\right)$, where

$$
A \sim S_{\alpha / 2}\left((\cos \pi \alpha / 4)^{2 / \alpha}, 1,0\right),
$$

and $\mathbf{G}=\left[G_{1}, \ldots, G_{d}\right]^{T} \sim \mathcal{N}\left(\mathbf{0}, \sigma^{2} \mathbf{I}\right)$ is called a subGaussian $\alpha$-stable random vector in $\mathbb{R}^{d}$ with underlying Gaussian vector $\mathbf{G}$.

Sub-Gaussian $\alpha$-stable random vectors also play an important role in studying complex $\alpha$-stable random variables; that is, a random variable with $\alpha$-stable distributed real and imaginary components. In particular, the generalization of symmetric $\alpha$-stable random variables to the complex case is known as the class of isotropic $\alpha$-stable random variables, defined as follows.

Definition 2. Let $Z_{1}, Z_{2}$ be two symmetric $\alpha$-stable random variables. The complex $\alpha$-stable random variable $Z=Z_{1}+$ $i Z_{2}$ is isotropic if it satisfies the condition

$$
e^{i \phi} Z \stackrel{(d)}{=} Z \text { for any } \phi \in[0,2 \pi) .
$$

Due to the fact that baseband signals are typically complex, isotropic $\alpha$-stable random variables will play an important role in the interference characterization.

The following proposition [19, Corollary 2.6.4] highlights the link between isotropic $\alpha$-stable random variables and subGaussian $\alpha$-stable random vectors.

Proposition 1. Let $0<\alpha<2$. A complex $\alpha$-stable random variable $Z=Z_{1}+i Z_{2}$ is isotropic if and only if there are two independent and identically distributed zero-mean Gaussian random variables $G_{1}, G_{2}$ with variance $\sigma^{2}$ and a random variable $A \sim S_{\alpha / 2}\left((\cos (\pi \alpha / 4))^{2 / \alpha}, 1,0\right)$ independent of $\left(G_{1}, G_{2}\right)^{T}$ such that $\left(Z_{1}, Z_{2}\right)^{T}=A^{1 / 2}\left(G_{1}, G_{2}\right)^{T}$. That is, $\left(Z_{1}, Z_{2}\right)^{T}$ is a sub-Gaussian $\alpha$-stable random vector.

We remark that isotropic complex $\alpha$-stable random variables are closely related to sub-Gaussian random vectors as can be observed from a comparison with Definition 1. Moreover, unlike the isotropic (or circularly symmetric) Gaussian case $(\alpha=2)$, isotropic $\alpha$-stable random variables with $\alpha<2$ do not have independent real and imaginary components. This dependence arises from the characterization in Proposition 1 through the dependence of the $\alpha$-stable random variable $A$ in both the real and imaginary components.

\section{B. Characterization of Interference Marginal Distributions}

The marginal distributions of the interference are characterized in the following theorem.

Theorem 1. Suppose that $h_{j, k}(t) x_{j, k}(t)$ is an isotropic complex random variable and

$$
\mathbb{E}\left[\left|\operatorname{Re}\left(h_{j, k}(t) x_{j, k}(t)\right)\right|^{4 / \eta} \mid\right]<\infty .
$$

\footnotetext{
${ }^{2}$ There exist also sub-Gaussian stable vectors allowing for more general dependence structure [19], but are not necessary for the purposes of this paper.
}

Then, $z_{k}(t)$ in (3) converges almost surely to an isotropic $4 / \eta$ stable random variable.

Moreover, if the fading coefficients $h_{j, k}(t)$ are i.i.d. and baseband emissions $x_{j, k}(t)$ are also i.i.d. then the scale parameters of all real and imaginary components are equal, given by

$$
\sigma_{y_{k}(t)}=\left(\pi \lambda p C_{\frac{4}{\eta}}^{-1} \mathbb{E}\left[\left|\operatorname{Re}\left(h_{j, k}(t) x_{j, k}(t)\right)\right|^{\frac{4}{\eta}}\right]\right)^{\frac{\eta}{4}},
$$

where

$$
C_{\alpha}= \begin{cases}\frac{1-\alpha}{\Gamma(2-\alpha) \cos (\pi \alpha / 2)}, & \text { if } \alpha \neq 1 \\ 2 / \pi, & \text { if } \alpha=1 .\end{cases}
$$

Proof. We sketch the proof as follows. By the mapping theorem for homogeneous Poisson point processes, the process $\left\{r_{j}^{2}\right\}$ is a one-dimensional Poisson point process with intensity $\pi \lambda p$. By an application of the LePage series representation of symmetric $\alpha$-stable random variables, it follows that $z_{k}(t)$ converges almost surely to

$$
z_{k}(t)=Z_{r}+i Z_{i}
$$

where $Z_{r}, Z_{i}$ are symmetric $4 / \eta$-stable random variables. Finally, $z_{k}(t)$ is isotropic due to the hypothesis that each $h_{j, k}(t) x_{j, k}(t)$ is an isotropic complex random variable. By Definition 2, it then follows that $z_{k}(t)$ is an isotropic complex $4 / \eta$-stable random variable.

We remark that the condition in Theorem 1 is satisfied for most common models for fading and baseband emissions. In particular, if $h_{j, k}(t)$ is Rayleigh and $x_{j, k}(t)$ is Gaussian then the condition is satisfied.

\section{Copula Interference Model}

As shown in (1), copulas provide a parameteric representation of the joint distribution for a random vector. As a consequence of Sklar's theorem [10], any joint distribution function admits a copula $C$. This copula is also unique if the marginal distributions are continuous, which is the case for the $\alpha$-stable marginals obtained in Section III-B.

Nevertheless, obtaining the exact copula is a challenging problem, both in its derivation and also for efficient parameter estimation and simulation. As such, we seek a copula that captures the joint dependence in the interference random vector $\mathbf{Z}$ in (6). This copula is required to:

1) well-approximate the true joint distribution of the interference random vector $\mathbf{Z}$;

2) and have a closed-form representation or be computationally feasible to numerically evaluate.

A good candidate for the copula to approximate $\mathbf{Z}$ can be obtained from standard parametric classes of copulas; namely, archimedean and $t$-copulas. As will be shown by a numerical study in Section III-D, the $t$-copula is particularly effective.

To this end, we recall the following definition of the $t$-copula [10]. Let $F_{v}$ be the distribution function of the univariate $t$ distribution, given by

$$
F_{v}(x)=\int_{-\infty}^{x} \frac{\Gamma\left(\frac{v+1}{2}\right)}{\sqrt{v \pi} \Gamma\left(\frac{v}{2}\right)}\left(1+\frac{t^{2}}{v}\right)^{-\frac{v+1}{2}} \mathrm{~d} t
$$


parameterized by the degree of freedom $v \in \mathbb{N}$. Moreover, the joint distribution $F_{v, \Sigma}$ of a $n$-dimensional multivariate $t$ distribution is given by

$$
F_{v, \Sigma}(\mathbf{x})=\int_{-\infty}^{x_{1}} \cdots \int_{-\infty}^{x_{n}} \frac{\Gamma\left(\frac{v+d}{2}\right)}{\Gamma\left(\frac{v}{2}\right) \sqrt{(\pi v)^{d}|\Sigma|}}\left(1+\frac{\mathbf{t}^{T} \Sigma^{-1} \mathbf{t}}{v}\right)^{-\frac{v+d}{2}} \mathrm{~d} \mathbf{t},
$$

parameterized by the degree of freedom $v \in \mathbb{N}$ and the $n \times n$ correlation matrix $\Sigma$. The $t$ copula is then defined as

$$
\left.C_{v, \Sigma}^{t}(\mathbf{u})=F_{v, \Sigma}\left(F_{v}^{-1}\left(u_{1}\right), \ldots, F_{v}^{-1}\left(u_{n}\right)\right)\right) .
$$

\section{Numerical Results}

We now study the quality of the $t$-copula model by fitting it to a data set obtained via Monte Carlo simulations. The Monte Carlo simulations implement the system model described in Section II. Recall that the system consists of a network of interfering devices. Each of these devices transmits on each band $k=1, \ldots, K$, with a probability $p>0$. The quantity $p K$ can be interpreted as the average quantity of frequency resources used by each device per transmission.

Our goal is to obtain a statistical model of the interference that fits the simulated data well in terms of the KL divergence. In all the numerical results, the KL divergence between the copula model and the simulated data set is obtained using the Information Theoretical Estimators (ITE) toolbox [20].

In the experiments, we compare three models all with $\alpha$ stable marginal distributions motivated by Theorem 1:

1) The $t$-copula model detailed in Section III-C.

2) The 2-dim. sub-Gaussian model consisting of independent two-dimensional sub-Gaussian random vectors. In this model, the $2 K$-dimensional random interference vector $\mathbf{Z}$ is decomposed into $K$ two-dimensional random vectors (corresponding to the real and imaginary parts of each band). Each two-dimensional random vector is sub-Gaussian (see Definition 1), independent from each of the other $K-1$ two-dimensional random vectors. This model is exact when interfering devices only transmit on a single band.

3) The $2 K$ sub-Gaussian model consisting of a $2 K$ dimensional sub-Gaussian random vector. This model corresponds to the scenario where all devices transmit on every band in $\mathcal{B}$, i.e., $p=1$ (see [7, Theorem 3]).

\section{TABLE I}

VALUE OF $p$ GIVING THE LARGEST KL DiVERGENCE BETWEEN THE $t$-COPULA MODEL AND 2-DIM. SUB-GAUSSIAN MODEL FOR DIFFERENT NUMBERS OF BANDS.

\begin{tabular}{|l|l|l|l|l|l|l|l|}
\hline$K$ & 2 & 3 & 4 & 5 & 6 & 7 & 8 \\
\hline \hline$p^{*}$ & 0.692 & 0.634 & 0.576 & 0.519 & 0.469 & 0.425 & 0.388 \\
\hline$D_{K L}$ & 0.136 & 0.192 & 0.221 & 0.247 & 0.275 & 0.287 & 0.290 \\
\hline
\end{tabular}

In Fig. 1 and 2, we plot the KL divergence, denoted as $D_{K L}$, of the three models with varying $p$ in 2 and 8 bands, respectively. As expected, the 2-dim. sub-Gaussian model has a very low KL divergence for $p \approx 0$ and the $2 K$ sub-Gaussian model has a very low KL divergence for $p \approx 1$. We also

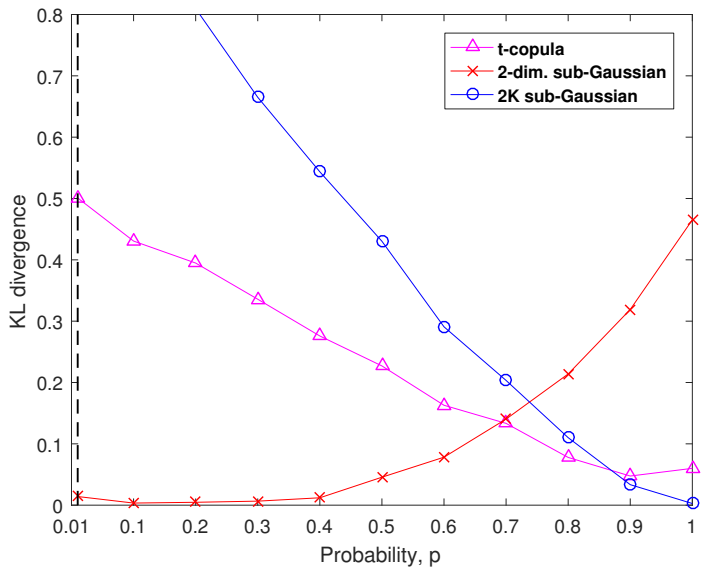

Fig. 1. Plot of the KL divergence between the simulated data set and three statistical models: $t$-copula model; 2 -dim. sub-Gaussian model; and $2 \mathrm{~K}$ subGaussian model. In the figure, $K=2$ frequency bands are considered.

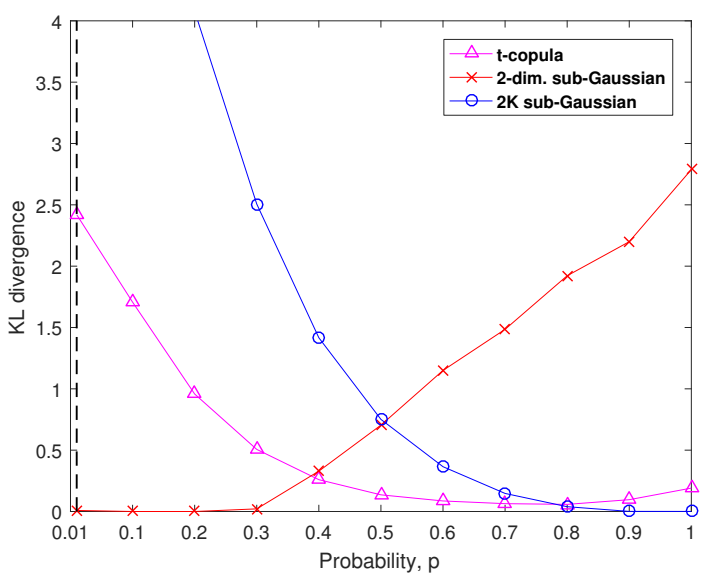

Fig. 2. Plot of the KL divergence between the simulated data set and three statistical models: $t$-copula model; 2 -dim. sub-Gaussian model; and $2 K$ subGaussian model. In the figure, $K=8$ frequency bands are considered.

observe that for $p>0.7$ ( $K=2$ bands) and $p>0.4$ ( $K=8$ bands), the $t$-copula model performs better than or comparably to the other two models. This suggests that a useful model for the interference statistics is to adopt the 2-dim. sub-Gaussian model for small $p$ and the $t$-copula model for larger $p$.

We also observe that as the number of bands $K$ varies, the value of $p$ giving the largest divergence between the $t$-copula model and the 2-dim. sub-Gaussian model (denoted by $p^{*}$ ) varies, illustrated in Table I. In particular, as $K$ increases, $p^{*}$ decreases. This suggests that for larger numbers of bands $K$, the $t$-copula model is the best model for a wider range of $p$.

TABLE II

KL DIVERGENCE BETWEen SIMUlated Data SET AND GaUSSian Model

\begin{tabular}{|l|l|l|l|l|l|l|}
\hline $\mathrm{p}$ & 0.01 & 0.1 & 0.2 & 0.3 & 0.4 & 0.5 \\
\hline $\mathrm{KL}$ & 111.82 & 105.19 & 91.32 & 102.91 & 92.87 & 95.63 \\
\hline $\mathrm{p}$ & 0.6 & 0.7 & 0.8 & 0.9 & 1 & \\
\hline $\mathrm{KL}$ & 97.73 & 86.60 & 96.41 & 96.09 & 89.38 & \\
\hline
\end{tabular}


Finally, note that as $K$ increases, the KL divergence at $p^{*}$ very slowly increases. This suggests that using the 2-dim. subGaussian model combined with the $t$-copula model has an error that is nearly independent of the dimension. We also make the same comparison with the Gaussian model in Table II., which shows that it is a poor model.

\section{INFORMATION THEORETIC LIMITS}

In this section, we study achievable rates of a point-topoint communication system described in Section II with interference governed by the copula model in Section III.

\section{A. Optimization Problem for the Capacity}

Motivated by the model developed in Section III, the joint distribution of the interference in (3) is of the form

$$
F_{\mathbf{Z}}\left(z_{1, r}, z_{1, i}, \ldots, z_{K, r}, z_{K, i}\right)=C\left(F_{1, r}\left(z_{1, r}\right), \ldots, F_{K, i}\left(z_{K, i}\right)\right),
$$

where $C$ is a copula.

At present, no bounds on the capacity for power-constrained inputs in general $\alpha$-stable noise channels are known [6], [7], [21]. As such, we follow [6], [7] and consider the constraints

$$
\begin{aligned}
\mathbb{E}\left[|\operatorname{Re}(X)|^{r}\right] & \leq c \\
\mathbb{E}\left[|\operatorname{Im}(X)|^{r}\right] & \leq c \\
|\operatorname{Re}(X)| & \leq A \\
|\operatorname{Im}(X)| & \leq A,
\end{aligned}
$$

where $0<r<\alpha, c>0$ and $A>0$. Note that although a power constraint is not explicitly imposed, the constraints on the amplitude imply that the power is finite.

Following the standard procedure used to study Gaussian noise channels, we relax the amplitude constraints, which via the noisy channel coding theorem [22] leads to the following optimization problem for the capacity

$$
\begin{aligned}
& \mathcal{C}=\sup _{\mu \in \mathcal{P}\left(\mathbb{R}^{2 K}\right)} I(\mathbf{X} ; \mathbf{Y}) \\
& \text { subject to } \mathbb{E}_{\mu}\left[|\mathbf{X}|^{r}\right] \preceq \mathbf{c},
\end{aligned}
$$

where $\mathcal{P}\left(\mathbb{R}^{2 K}\right)$ corresponds to the set of probability measures on $\left(\mathbb{R}^{2 K}, \mathcal{B}\left(\mathbb{R}^{2 K}\right)\right)$.

\section{B. Achievable Rates}

The following theorem gives achievable rates for the channel in (5).

Theorem 2. An achievable rate for the channel in (4) subject to the constraints in (20) is given by

$$
\mathcal{C} \geq R=\frac{1}{\alpha} \log \left(1+\left(|a|_{\min } \frac{\sqrt[r]{\frac{c}{C(r, \alpha)}}}{\sigma_{\mathbf{Z}}}\right)^{\alpha}\right),
$$

where

$$
C(r, \alpha)=\frac{2^{r+1} \Gamma\left(\frac{r+1}{2}\right) \Gamma(-r / \alpha)}{\alpha \sqrt{\pi} \Gamma(-r / 2)} .
$$

and $|a|_{\min }=\min \left\{\left|a_{i i}\right|, \quad i=1, \ldots, 2 K\right\}$ with $a_{i i}$ the $i$-th diagonal element of $\mathbf{A}, \sigma_{\mathbf{Z}}$ is the scale parameter of each element of $\mathbf{Z}$, given by (12).
Proof. By Theorem 1, the scale parameters of each component of the noise vector $\mathbf{Z}$ in (5) are equal. Therefore, there exists a random vector $\mathbf{W}$ with $\alpha$-stable marginals with unit scale parameters and constant $\sigma_{\mathbf{Z}} \in \mathbb{R}_{+}$given in (12) such that

$$
\mathbf{Z} \stackrel{d}{=} \sigma_{\mathbf{Z}} \mathbf{W} .
$$

By [23], the fractional moment of a symmetric $\alpha$-stable random variable $V$ with scale parameter $\sigma_{V}$ is given by

$$
\mathbb{E}\left[|V|^{r}\right]=C(r, \alpha) \sigma_{V}^{r},
$$

where

$$
C(r, \alpha)=\frac{2^{r+1} \Gamma\left(\frac{r+1}{2}\right) \Gamma(-r / \alpha)}{\alpha \sqrt{\pi} \Gamma(-r / 2)} .
$$

As such, recalling that $\mathbf{A}$ is a diagonal matrix and choosing $\mathbf{X} \stackrel{d}{=} \sigma_{\mathbf{X}} \mathbf{A}^{-1} \mathbf{X}_{I}$, where $\mathbf{X}_{I}$ is a random vector with the same distribution as $\mathbf{W}$,

$$
\sigma_{\mathbf{X}}=|a|_{\min } \sqrt[r]{\frac{c}{C(r, \alpha)}}
$$

and $|a|_{\min }=\min \left\{\left|a_{i i}\right|, \quad i=1, \ldots, 2 K\right\}$ with $a_{i i}$ the $i$-th diagonal element of $\mathbf{A}$. This ensures the constraint in (20) is satisfied.

By the stability property of scalar symmetric $\alpha$-stable random variables,

$$
\begin{aligned}
\mathbf{Y} & :=\left(\sigma_{\mathbf{X}}^{\alpha}+\sigma_{\mathbf{Z}}^{\alpha}\right)^{\frac{1}{\alpha}} \mathbf{V} \\
& =\sigma \mathbf{V}
\end{aligned}
$$

where $\mathbf{V}$ is a random vector with symmetric $\alpha$-stable marginals, each with scale parameter 1 .

Let $X_{1}, X_{I, 1}, W_{1}$ be the first elements of $\mathbf{X}, \mathbf{X}_{I}, \mathbf{W}$, respectively. Then,

$$
\begin{aligned}
I(\mathbf{X} ; \mathbf{Y}) & =I(\mathbf{X} ; \mathbf{A X}+\mathbf{Z}) \\
& \geq I\left(X_{1} ; \sigma\left(\frac{a_{1} X_{1}}{\sigma}+\frac{Z_{1}}{\sigma}\right)\right) \\
& =I\left(X_{1} ; \sigma\left(\frac{\sigma_{\mathbf{X}}}{\sigma} X_{I, 1}+\frac{\sigma_{\mathbf{Z}}}{\sigma} W_{1}\right)\right) \\
& =\log \frac{\sigma}{\sigma_{\mathbf{Z}}}+h\left(\frac{\sigma_{\mathbf{X}}}{\sigma} X_{I, 1}+\frac{\sigma_{\mathbf{Z}}}{\sigma} W_{1}\right)-h\left(W_{1}\right) \\
& =\log \frac{\sigma}{\sigma_{\mathbf{Z}}}
\end{aligned}
$$

where the inequality follows from the chain rule for mutual information [24]. The last equality follows from the fact that $X_{I, 1}$ and $W_{1}$ are symmetric $\alpha$-stable random variables with unit scale parameters. Therefore, the scale parameter of $\frac{\sigma_{\mathbf{X}}}{\sigma} X_{I, 1}+\frac{\sigma_{\mathbf{Z}}}{\sigma} W_{1}$ is $\left(\frac{1}{\sigma_{\mathbf{X}}^{\alpha}+\sigma_{\mathbf{Z}}^{\alpha}}\left(\sigma_{\mathbf{X}}^{\alpha}+\sigma_{\mathbf{Z}}^{\alpha}\right)\right)^{\frac{1}{\alpha}}=1$. Applying (24) to (28) then yields the desired result.

\section{Numerical Results}

Through the scale parameter $\sigma_{\mathbf{Z}}$, the achievable rates in Theorem 2 implicitly depend on the probability that a given device access a given band, and also the intensity $\lambda$ of the Poisson point process governing the locations of the interferers. In particular, we observe that when all other model parameters-including $\alpha, \eta$ and the fractional moments of the 
fading and base-band emmisions - are constant, the achievable rate is proportional to $\log \left(1+\frac{Q}{\lambda p}\right)$, where $Q$ is a constant depending on the remaining fixed model parameters.

In Fig. 3, we plot the achievable rate in (21) with varying probability $p$ a given user accesses a given band. Observe that the achievable rate decreases as $p$ increases. The main reason for this is that as $p$ increases, the scale parameter of the interference also increases. We remark that in the achievable rate in (21), the copula dependence structure of the interference does not explicitly appear. We note however that it does appear in the distribution of the input $\mathbf{X}$.

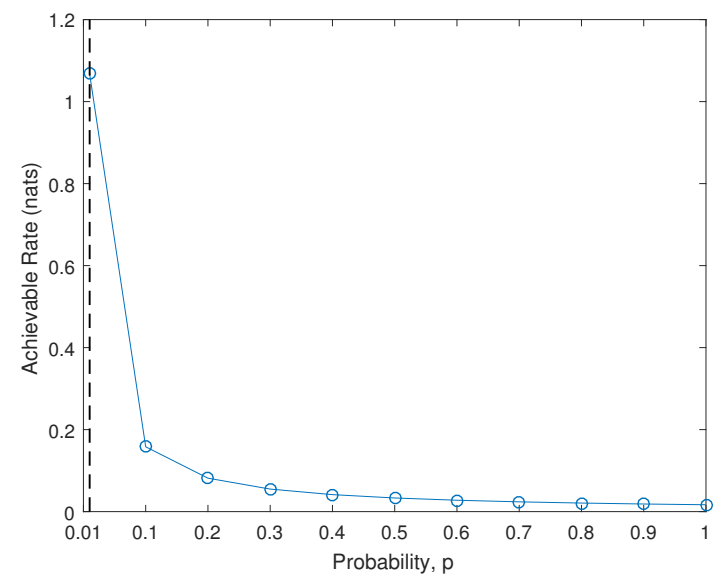

Fig. 3. Plot of the lower bound achievable rate and area spectral efficiency

\section{CONCLUSIONS}

A basic challenge in communication over the ISM bands is that a number of large-scale IoT technologies have been proposed that have significantly different protocols. These differences lead to non-Gaussian interference and, in systems transmitting over multiple frequency bands, dependence between the interference on each band.

To provide tractable yet accurate models for the interference, we have proposed interference models based on the $t$ copula and $\alpha$-stable marginals. This model has been validated on a simulated data set, which exhibits non-trivial dependence structures.

To illustrate the tractability of the copula interference model, we considered a point-to-point communication link in the presence of interference from an IoT system. We derived a closed-form expression for achievable rates. These results provide a basis to study resource allocation and network design, which we intend to explore in future work.

\section{ACKNOWLEDGEMENTS}

This work has been (partly) funded by the French National Agency for Research (ANR) under grant ANR-16-CE25-0001 - ARBURST.

\section{REFERENCES}

[1] M. Lauridsen, B. Vejlgaard, I. Kovács, H. Nguyen, and P. Mogensen, "Interference measurements in the European $868 \mathrm{MHz}$ ISM band with focus on LoRa and SigFox," in IEEE Wireless Communications and Networking Conference (WCNC), 2017.

[2] A. Moro, "Potential interference generated by UHF RFID systems on military telecommunication devices," Italian National Agency for New Technologies, vol. 3, 2007

[3] M. Simek, M. Fuchs, L. Mraz, P. Moravek, and M. Botta, "Measurement of LowPAN network coexistence with home microwave appliances in laboratory and home environments," Broadband and Wireless Computing, Communication and Applications, vol. 10, pp. 292-299, 2011

[4] B. Vejlgaard, M. Lauridsen, H. Nguyen, I. Kovács, P. Mogensen, and M. Sørensen, "Interference impact on coverage and capacity for low power wide area IoT networks," in IEEE Wireless Communications and Networking Conference (WCNC), 2017.

[5] P. Pinto and M. Win, "Communication in a Poisson field of interfererspart I: interference distribution and error probability," IEEE Transactions on Wireless Communications, vol. 9, no. 7, pp. 2176-2186, 2010.

[6] M. Egan, L. Clavier, M. de Freitas, L. Dorville, J.-M. Gorce, and A. Savard, "Wireless communciation in dynamic interference," in IEEE Global Communciations Conference (GLOBECOM), 2017.

[7] M. Egan, L. Clavier, C. Zheng, M. de Freitas, and J.-M. Gorce, "Dynamic interference for uplink SCMA in large-scale wireless networks without coordination," EURASIP Journal on Wireless Communications and Networking, vol. 1, 2018.

[8] L. Dai, B. Wang, Y. Yuan, S. Han, C.-L. I, and Z. Wang, "Nonorthogonal multiple access for 5G: solutions, challenges, opportunities, and future research trends," IEEE Communications Magazine, vol. 53, no. 9 , pp. 74-81, 2015.

[9] H. Nikopour and H. Baligh, "Sparse code multiple access," in IEEE International Symposium on Personal Indoor and Mobile Radio Communications (PIMRC), 2013.

[10] R. Nelson, An Introduction to Copulas. New York, NY: Springer-Verlag, 1999.

[11] M. Gholizadeh, H. Amindavar, and J. Ritcey, "On the capacity of MIMO correlated Nakagami-m fading channels using copula," EURASIP Journal on Wireless Communications and Networking, vol. 1, 2015.

[12] J. Kitchen and W. Moran, "Copula techniques in wireless communications," ANZIAM Journal, vol. 51, pp. 526-540, 2010.

[13] X. Yan, L. Clavier, G. Peters, N. Azzaoui, F. Septier, and I. Nevat, "Skew-t copula for dependence modelling of impulsive (alpha-stable) interference," in IEEE International Conference on Communications (ICC), 2015.

[14] E. Soret, L. Clavier, G. Peters, F. Septier, and I. Nevat, "Modeling interference with alpha-stable distributions and copulae for receiver design in wireless communications," in Proceedings of 61st ISI World Statistics Congress, 2017.

[15] J. Ilow and D. Hatzinakos, "Analytic alpha-stable noise modeling in a poisson field of interfers or scatterers," IEEE Transactions on Signal Processing, vol. 46, no. 6, pp. 1601-1611, 1998.

[16] X. Yang and A. Petropulu, "Co-channel interference modeling and analysis in a Poisson field of interferers in wireless communications," IEEE Transactions on Signal Processing, vol. 51, no. 1, pp. 64-76, 2003.

[17] K. Gulati, B. Evans, J. Andrews, and K. Tinsley, "Statistics of co-channel interference in a field of Poisson-Poisson clustered interferers," IEEE Transactions on Signal Processing, vol. 58, no. 12, pp. 6207-6222, 2010.

[18] C. Nikias and M. Shao, Signal processing with alpha-stable distributions and applications. New York, NY: Wiley, 1995.

[19] G. Samorodnitsky and M. Taqqu, Stable Non-Gaussian Random Processes. New York, NY: CRC Press, 1994.

[20] Z. Szabó, "Information theoretical estimators toolbox," Journal of Machine Learning Research, vol. 15, pp. 283-287, 2014.

[21] M. de Freitas, M. Egan, L. Clavier, G. Peters, and N. Azzaoui, "Capacity bounds for additive symmetric alpha-stable noise channels," IEEE Transactions on Information Theory, vol. 63, pp. 5115-5123, Aug. 2017.

[22] T. Han, Information-Spectrum Methods in Information Theory. Berlin Heidelberg: Springer-Verlag, 2003.

[23] V. Zolotarev, "Mellin-stieltjes transforms in probability theory," Theory Probab. Appl., vol. 2, no. 4, pp. 433-460, 1957.

[24] T. Cover and J. Thomas, Elements of Information Theory, Second Edition. Hoboken, NJ: John Wiley \& Sons, Inc., 2006. 